\title{
Density-functional study of the Mott gap in the Hubbard model
}

\author{
N. A. Lima and L. N. Oliveira \\ Departamento de Física e Informática, Instituto de Física de São Carlos, Universidade de São Paulo, \\ Caixa Postal 369, 13560-970 São Carlos, SP, Brazil \\ K. Capelle \\ Departamento de Química e Física Molecular, Instituto de Química de São Carlos, Universidade de São Paulo, \\ Caixa Postal 780, São Carlos, 13560-970 SP, Brazil \\ accepted by Europhysics Letters (2002)
}

(November 20, 2018)

\begin{abstract}
We study the Mott insulating phase of the one-dimensional Hubbard model using a local-density approximation (LDA) that is based on the Bethe Ansatz (BA). Unlike conventional functionals the BA-LDA has an explicit derivative discontinuity. We demonstrate that as a consequence of this discontinuity the BA-LDA yields the correct Mott gap, independently of the strength of the correlations. A convenient analytical formula for the Mott gap in the thermodynamic limit is also derived. We find that in one-dimensional quantum systems the contribution of the discontinuity to the full gap is more important than that of the band-structure gap, and discuss some consequences this finding has for electronic-structure calculations.
\end{abstract}

71.15.Mb, 71.10.Pm, 71.10.Fd

The intricacies of the correlation-induced metalinsulator transition ('Mott transition') have fascinated and challenged physicists for many years, and a multitude of methods has been brought to bear on the problem [1]. Here we study the Mott insulator from the point of view of density-functional theory (DFT), a method whose full potential for exploring the Mott phenomenon has not been widely recognized. It may be particularly timely to study the prospects for a DFT treatment of the Mott insulating phase because this is one of the two main phases of the one-dimensional Hubbard model (1DHM), the paradigmatic model for low-dimensional quantum systems. The recent interest in nanotechnology has brought quasi one-dimensional systems, such as quantum wires and carbon nanotubes, into the focus of current research in many-body physics and materials science. DFT, on the other hand, is the de facto standard method for the calculation of the electronic structure of materials, but its applicability to the very peculiar phases typical of low-dimensional quantum systems has not been systematically investigated. Here we report on the application of a very recently developed density functional [2] to the Mott insulating phase of the 1DHM.

DFT is often applied in a single-particle mode, in which the eigenvalues of the Kohn-Sham (KS) equation are interpreted as a mean-field approximation to the quasiparticle energies. It is well known that this approximation breaks down for systems with strong correlations, of which the Mott insulator is an example. On the other hand, as long as one restricts oneself to working with the particle density, the total ground-state energy, and quantities derivable from these, DFT is a rigorous manybody theory, whose predictive power is only limited by the quality of available approximations for the exchangecorrelation $(x c)$ functional. It is in this many-body mode that we employ DFT here.

The choice of the appropriate approximation for the $x c$ functional depends on the system under study. Here we investigate the 1DHM, since this model represents a particularly clear case of a Mott transition, free of unessential complications: for nonzero interaction strength $(U>$ 0) the $1 \mathrm{DHM}$ is a Mott insulator at half filling, whereas it is a Luttinger liquid (i.e., a one-dimensional metal) at all other fillings [3-5]. Following pioneering work of Gunnarsson, Schönhammer, and Noack [6,7] we have recently constructed an approximate $x c$ functional for this model [2]. This functional is a local-density approximation (LDA) that is based on the exact Bethe Ansatz (BA) solution of the homogeneous $1 \mathrm{DHM}$ in the thermodynamic limit. For $N \leq 1$ it takes the form

$$
E_{x c}^{B A L D A}\left[n_{i}, U\right]=\sum_{i} e_{x c}^{<}\left(n_{i}, U\right),
$$

where $e_{x c}^{<}(n, U)=e^{<}(n, U)-e^{<}(n, 0)-U n^{2} / 4$ is the per-site $x c$ energy of the homogeneous infinite 1DHM, and $e^{<}(n, U)$ is the corresponding total energy, for which we have given an accurate parametrization, based on the Bethe Ansatz, in Ref. [2]. For $n_{i}>1$ a particle-hole transformation shows that $e_{x c}^{<}(n, U)$ must be replaced by $e_{x c}^{>}(n, U)=e_{x c}^{<}(2-n, U)$. Here we apply this functional to the Mott insulator.

Our starting point is the exact expression for the energy gap of a many-body system, $\Delta=I-A$, where $I=E(N-1)-E(N)$ is the system's ionization energy, $A=E(N)-E(N+1)$ its electron affinity, and $E(N)$ the ground-state energy of the $N$-particle system. Hence, 


$$
\Delta=E(N+1)+E(N-1)-2 E(N) .
$$

In ensemble DFT one can derive an alternative, but equivalent, representation for the true energy gap, namely [8]

$$
\Delta=\epsilon_{k_{\max }+1}(N)-\epsilon_{k_{\max }}(N)+\Delta_{x c}=: \Delta_{K S}+\Delta_{x c},
$$

where $\epsilon_{k}(N)$ is the $k^{\prime}$ th single-particle energy (KS eigenvalue) of the $N$-particle system, and $\Delta_{x c}$ is the discontinuity of the exchange-correlation potential [9]

$$
\Delta_{x c}=\left.\frac{\left.\delta E_{x c}[n]\right]}{\delta n(\mathbf{r})}\right|_{N+\delta}-\left.\frac{\left.\delta E_{x c}[n]\right]}{\delta n(\mathbf{r})}\right|_{N-\delta},
$$

where $\delta \rightarrow 0^{+}$is an infinitesimal change in the total particle number $N$. According to ensemble DFT, for open systems the total energy for fractional particle number is given by a straight-line interpolation between the energies obtained for integer particle number [9]. It is this fact that guarantees the equivalence of both representations [8].

For the homogeneous infinite 1DHM at $n=1$ one can evaluate both Eq. (2) and Eq. (3) analytically from the parametrization underlying the BA-LDA. In either case we find

$$
\Delta_{h o m}^{B A L D A}(U)=U+4 \cos \left(\frac{\pi}{\beta(U)}\right),
$$

where $\beta(U)$ is calculated from [2]

$$
-\frac{2 \beta}{\pi} \sin \left(\frac{\pi}{\beta}\right)=-4 \int_{0}^{\infty} d x \frac{J_{0}(x) J_{1}(x)}{x[1+\exp (U x / 2)]},
$$

and $J_{0}$ and $J_{1}$ are zero and first order Bessel functions. (Here and below we take $t=1$ as our unit of energy.)

Representation (3) shows that there are in general two contributions to the many-body gap, one being the KS gap, $\Delta_{K S}$ (this is the contribution obtained in a standard band-structure calculation), the other the correlationinduced Mott gap, $\Delta_{x c}$, which is a pure many-body effect. Unfortunately, the most commonly employed approximate $x c$ functional, the conventional ab initio LDA, does not have a discontinuity at integer $N$, so that $\Delta_{x c}^{L D A}=0$ and $\Delta^{L D A}=\Delta_{K S}^{L D A}$. This is the origin of the well known band-gap problem of the LDA [9]. The same applies to common gradient-corrected functionals. In the case of $a b$ initio calculations one finds it thus necessary to reach beyond DFT and employ more or less systematically derived correction terms, such as the scissors operator [10], LDA+U [11], or LDA+SIC [12]. However, the situation is different for the BA-LDA. Due to the particle-hole transformation involved in its construction this functional naturally has a discontinuity at half filling (occupation number $n=1$ ), where the underlying homogeneous infinite 1DHM undergoes its metal-insulator transition. The BA-LDA is thus uniquely suited to test the density-functional theory of the many-body energy gap.

To begin our analysis we present, in the left half of Fig. 1, the energy gap of a homogeneous 1 DHM with $L=10$ sites, calculated with four different approaches: (i) BA-LDA gap calculated numerically from representation (2), (ii) energy gap obtained from representation (2) if the pseudo LDA of Refs. [6,13] (GS-LDA) is used instead of the BA-LDA (note that that functional does not have a discontinuity), (iii) BA-LDA gap calculated analytically for an infinite homogeneous system from Eq. (5), and (iv) the exact gap, obtained by numerical diagonalization. Clearly, the continuous functional drastically underestimates the exact gap. In the right half of Fig. 1 we present the same results for a 150 -site system. The last column is now missing since a system of this size cannot be treated anymore by numerical exact diagonalization. However, precisely because $L$ is large, we now observe that the gap obtained numerically from the BA-LDA (the first bar) is close to the analytical result of Eq. (5) (third bar).

The main result that follows from the data presented in Fig. 1 is that for $U=6$ the dominating contribution to the many-body gap is due to the discontinuity. We have repeated the same analysis also for other values of $U$ and $L$, and found that the critical value of $U$ for which the discontinuity gap $\Delta_{x c}$ becomes more important than the band-structure gap $\Delta_{K S}$ decreases rapidly with increasing $L$, e.g. $U_{c}(L=6)=5.2$ and $U_{c}(L=10)=4.2$, while $U_{c}(L=500)=1.8$. For large systems and realistic values of $U$ the discontinuity is thus the dominating contribution to the full gap. This observation is in agreement with that of Refs. [7] and [14], but contradicts the one of Ref. [13].

From our results it follows that the discontinuity contribution is absolutely crucial for obtaining the correct many-body gap from DFT. This implies that in order to solve the band gap problem encountered in the DFT description of semiconductors and insulators it is not useful to optimize a continuous functional (e.g. by passing from $L D A$ to GGA) or to try to improve on the band-structure part of the calculation (which will only result in an improved value for $\Delta_{K S}$ ); rather one should search for a properly discontinuous functional. Interestingly, Ref. [15] reports quantitative agreement of the KS gap calculated from the B3LYP GGA functional and the experimental gap for a variety of semiconductors and insulators. These authors themselves conclude that this agreement is probably fortuitious - an interpretation that is confirmed by the present analysis.

Fig. 2 displays, for a 150-site system, the BA-LDA [2] gap and the pseudo-LDA $[6,13]$ gap, both calculated numerically from representation (2) as a function of the interaction $U$. The two full curves are asymptotic results (valid in the thermodynamic limit) that can be extracted analytically from the Bethe Ansatz solution of the 1DHM 
in the limits $U \rightarrow \infty$ and $U \rightarrow 0$, respectively. For $U \rightarrow \infty[5,16]$

$$
\Delta(U \rightarrow \infty)=U-4+(8 \ln 2) / U .
$$

The leading-order term in this expansion, $U-4$, is also obtained immediately from our Eq. (5), since for $U \rightarrow \infty$ one has $\beta=1$ [2]. For $U \rightarrow 0$, on the other hand [5,16],

$$
\Delta(U \rightarrow 0)=\frac{8}{\pi} \sqrt{U} \exp \left(-\frac{2 \pi}{U}\right) .
$$

For $U=0$ Eq. (8) goes to zero. This, too, is reproduced by Eq. (5), since for $U=0$ one has $\beta=2[2]$. However, the non-analyticity of the exact expression (8) is not properly accounted for by Eq. (5). This is not a defect of the LDA (which becomes exact in the thermodynamic limit of a homogeneous system), but of our parametrization of it, which does not incorporate this nonanalyticity. Work on improved parametrizations is in progress.

As clearly seen in Fig. 2, the BA-LDA reproduces the limiting behaviours quite well, both for large and for small $U$, in spite of the intrinsic error of the underlying parametrization. The pseudo LDA, on the other hand, is incapable of reproducing the exact gap for large $U$.

In Fig. 3 we study the evolution of the various contributions to the many-body gap as an originally infinite homogeneous system with $U=6$ is gradually made finite. Both the many-body gap and the single-particle gap scale approximately linearly with inverse system size. At $1 / L=0$, i.e., in the thermodynamic limit, the singleparticle gap is zero. A band-structure calculation would predict the system to be a metal in this limit. The $x c$ contribution to the gap, however, is nonzero and can be calculated from Eq. (5). For finite system sizes $(1 / L>0)$ the boundaries give rise to quantization of the singleparticle states and a discrete set of energy levels. The KS gap then becomes nonzero. The many-body gap is in this case calculated numerically from Eq. (2), and the $x c$ gap is extracted from that result by means of Eq. (3). As seen in the inset of Fig. 3, the $x c$ gap is a nearly quadratic function of $1 / L$, i.e., does not scale with the same power as the single-particle gap.

Interestingly, the $x c$ gap in the $1 \mathrm{DHM}$ is larger than the single-particle gap all the way from $L \rightarrow \infty$ down to $L \sim O(1)$. Applied to realistic quasi one-dimensional systems, such as quantum wires and nanotubes, this observation implies that the $x c$ gap is of paramount importance for nanophysics and device technology. Unfortunately, this contribution is completely missed by standard band-structure approaches, which only calculate the single-particle gap. Notable exceptions are the optimized-effective potential method [17-19] and the 'electron-gas with a gap' approach of Refs. [20-22]. In view of our findings such methods may aquire additional significance for first-principles calculations of the electronic structure of low-dimensional systems.
In summary, we have demonstrated the applicability of the BA-LDA approach to the Mott insulating phase of the 1DHM. We find that the discontinuity of the exchange-correlation functional makes a crucial contribution to the full gap, both for finite and for infinite systems, and for a wide range of values of $U$. A continuous functional drastically underestimates the exact gap, whereas the BA-LDA yields quantitatively reliable results with very modest computational effort. This establishes the BA-LDA, which was originally designed for the Luttinger-liquid phase [2], as a computational tool also in the insulating phase of the 1DHM. Although the BA-LDA was constructed specifically for the 1DHM and cannot be used directly with the $a b$ initio Hamiltonian, we believe our findings concerning the discontinuity to be robust and qualitatively valid also for ab initio calculations. In particular, we expect that also for $a b i n i$ tio calculations a reliable determination of the electronic structure of quantum wires and nanotubes can only be achieved if a functional with a proper discontinuity is employed.

Acknowledgments This work was supported by FAPESP and CAPES. We thank M. F. da Silva for many discussions and providing us with the exact diagonalization program. KC thanks E. K. U. Gross and A. Schindlmayr for helpful discussions.

[1] N. F. Mott, Metal-Insulator Transitions 2nd. ed. (Taylor \& Francis, London, 1990). F. Gebhard, The Mott MetalInsulator Transition, Springer Tracts in Modern Physics Vol. 137 (Springer, New York, 1997). M. Imada, A. Fujimori, and Y. Tokura, Rev. Mod. Phys. 70, 1039 (1998).

[2] N. A. Lima, M. F. Silva, L. N. Oliveira, and K. Capelle, submitted (2001) [cond-mat 0112428].

[3] E. H. Lieb and F. Y. Wu, Phys. Rev. Lett. 20, 1445 (1968).

[4] J. Voit, Rep. Prog. Phys. 58, 977 (1995).

[5] P. Schlottmann, Int. J. Mod. Phys. B 11, 355 (1997).

[6] O. Gunnarsson and K. Schönhammer, Phys. Rev. Lett. 56, 1968 (1986).

[7] K. Schönhammer, O. Gunnarsson, and R. M. Noack, Phys. Rev. B 52, 2504 (1995).

[8] R. M. Dreizler and E. K. U. Gross, Density Functional Theory (Springer-Verlag, Berlin, 1990).

[9] J. P. Perdew, R. G. Parr, M. Levy, and J. L. Balduz, Phys. Rev. Lett. 49, 1691 (1982). J. P. Perdew and M. Levy, Phys. Rev. Lett. 51, 1884 (1983). L. J. Sham and M. Schlüter, Phys. Rev. Lett. 51, 1888 (1983).

[10] G. Baraff and M. Schlüter, Phys. Rev. B 30, 3460 (1984).

[11] A. I. Liechtenstein, V. I. Anisimov, and J. Zaanen, Phys. Rev. B 52, 5467 (1995). V. I. Anisimov, J. Zaanen, and O. K. Andersen, Phys. Rev. B 44, 943 (1991).

[12] A. Svane and O. Gunnarsson, Phys. Rev. Lett. 651148 
(1990). Z. Szotek, W. M. Temmermann, and H. Winter, Phys. Rev. Lett. 721244 (1994).

[13] K. Schönhammer and O. Gunnarsson, J. Phys. C 20, 3675 (1987).

[14] R. W. Godby, M. Schlüter, and L. J. Sham, Phys. Rev. Lett. 56, 2415 (1986). M. Lannoo, M. Schlüter, and L. J. Sham, Phys. Rev. B 32, 3890 (1985).

[15] J. Muskat, A. Wander, and N. M. Harrison, Chem. Phys. Lett. 342, 397 (2001).

[16] A. A. Ovchinnikov, Sov. Phys. JETP 30, 1160 (1970).

[17] J. B. Krieger, Y. Li, and G. J. Iafrate, Phys. Rev. A 45, 101 (1992); Phys. Lett. A 146, 256 (1990).

[18] T. Kotani, Phys. Rev. Lett. 74, 2989 (1995).

[19] T. Grabo, T. Kreibich, S. Kurth, and E. K. U. Gross, in Strong Coulomb Correlations in Electronic Structure Calculations: Beyond the Local Density Approximation, Ed. V. I. Anisimov (Gordon \& Breach, 1999).

[20] C. Gutle, A. Savin, J. B. Krieger, and J. Q. Chen, Int. J. Quantum Chem. 75, 885 (1999).

[21] J. Rey and A. Savin, Int. J. Quantum Chem. 69, 581 (1998).

[22] S. Kurth, J. P. Perdew, and P. Blaha, Int. J. Quantum Chem. 75, 889 (1999).

FIG. 1. Many-body energy gap of the one-dimensional Hubbard model for $U=6$. Left: $L=10$ sites. First bar: calculated from the BA-LDA using Eq. (2). Second bar: calculated from the continuous pseudo LDA, again using Eq. (2). Third bar: calculated from the analytical BA-LDA expression (5), valid in the thermodynamic limit $L \rightarrow \infty$. Fourth bar: exact result. Right: same as left, but with the first two bars now calculated for a system with $L=150$ sites. As a consequence of the system size the bar with the exact result is missing.

FIG. 2. Energy gap calculated from Eq. (2) versus interaction $U$ for a homogeneous system with $L=150$ sites. Squares: BA-LDA, circles: pseudo LDA. The full and dashed curves are the asymptotically valid analytical results for $U \rightarrow \infty$ and $U \rightarrow 0$ of Eqs. (7) and (8), respectively.

FIG. 3. Energy gap of a finite $1 \mathrm{DHM}$ as a function of inverse system size $1 / L$. Upper curve (triangles): many-body gap $\Delta$, calculated from Eq. (2). Lower curve (circles): Kohn-Sham gap $\Delta_{K S}$, calculated as a difference of single-particle eigenvalues. Middle curve (squares): $x c$ gap $\Delta_{x c}$, calculated as the difference between the two other curves [i.e., from Eq. (3)]. Inset: $x c$ gap on a smaller scale, showing approximately parabolic behaviour of $\Delta_{x c}$ as a function of $1 / L$. 

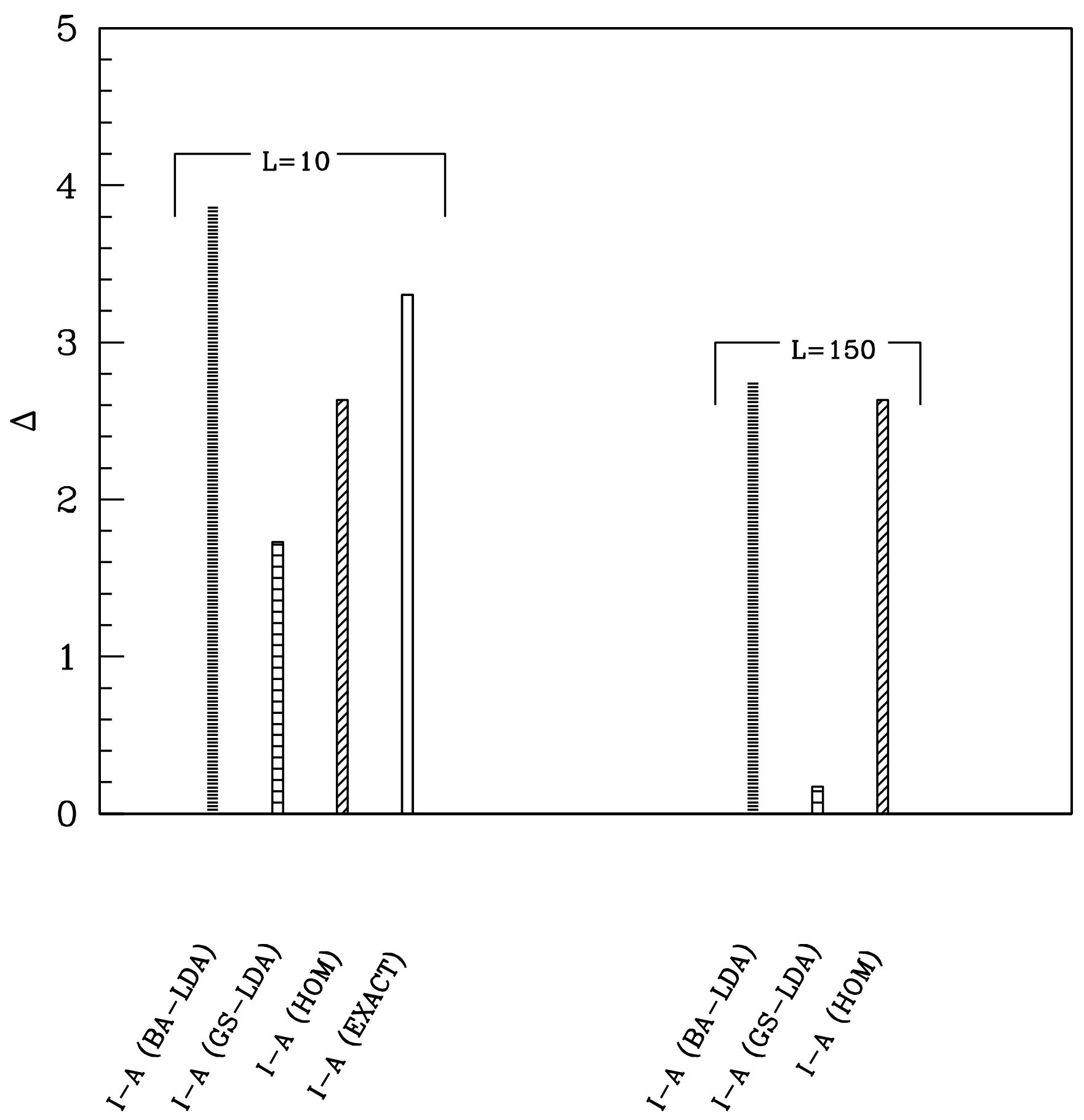


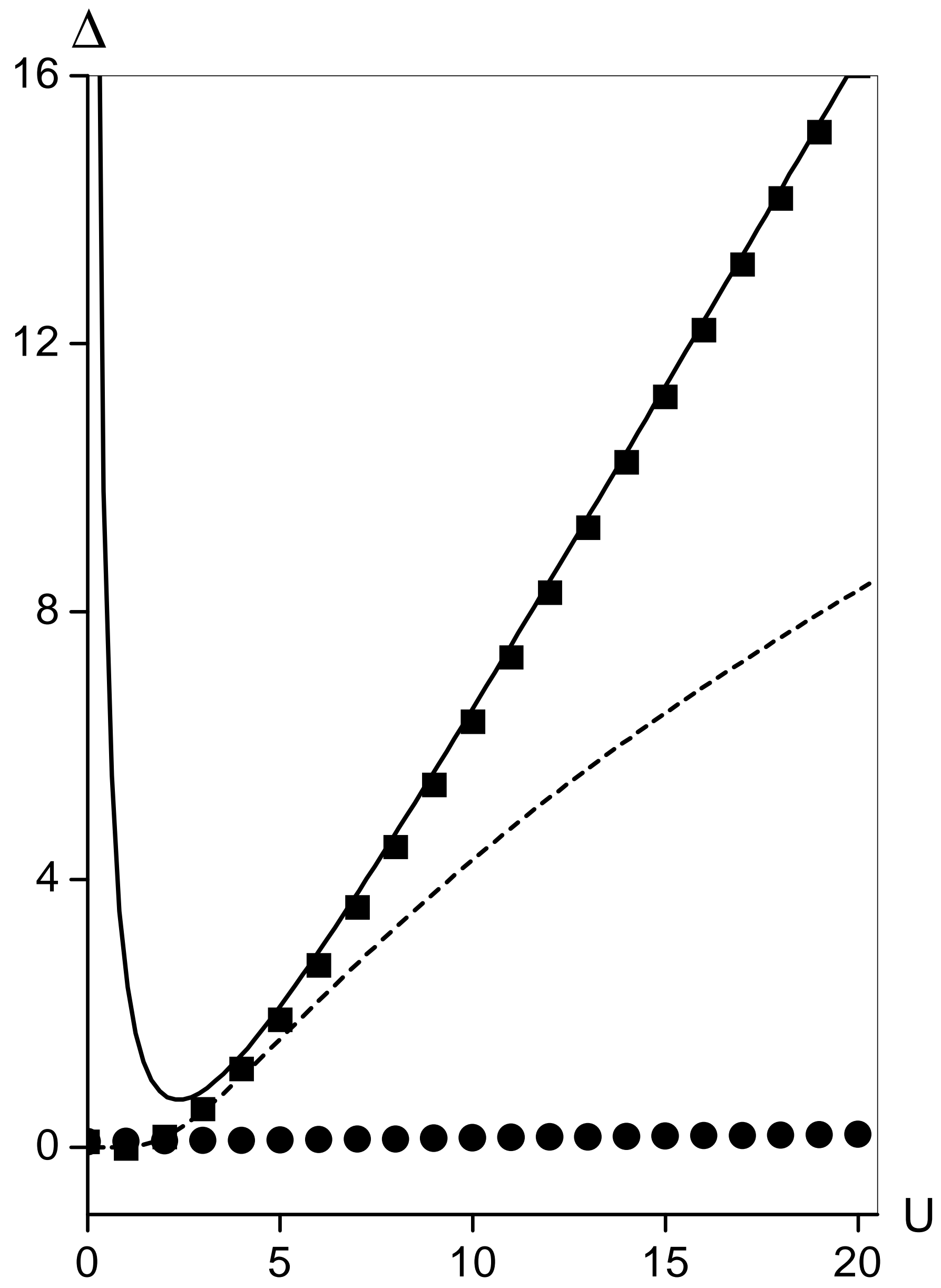




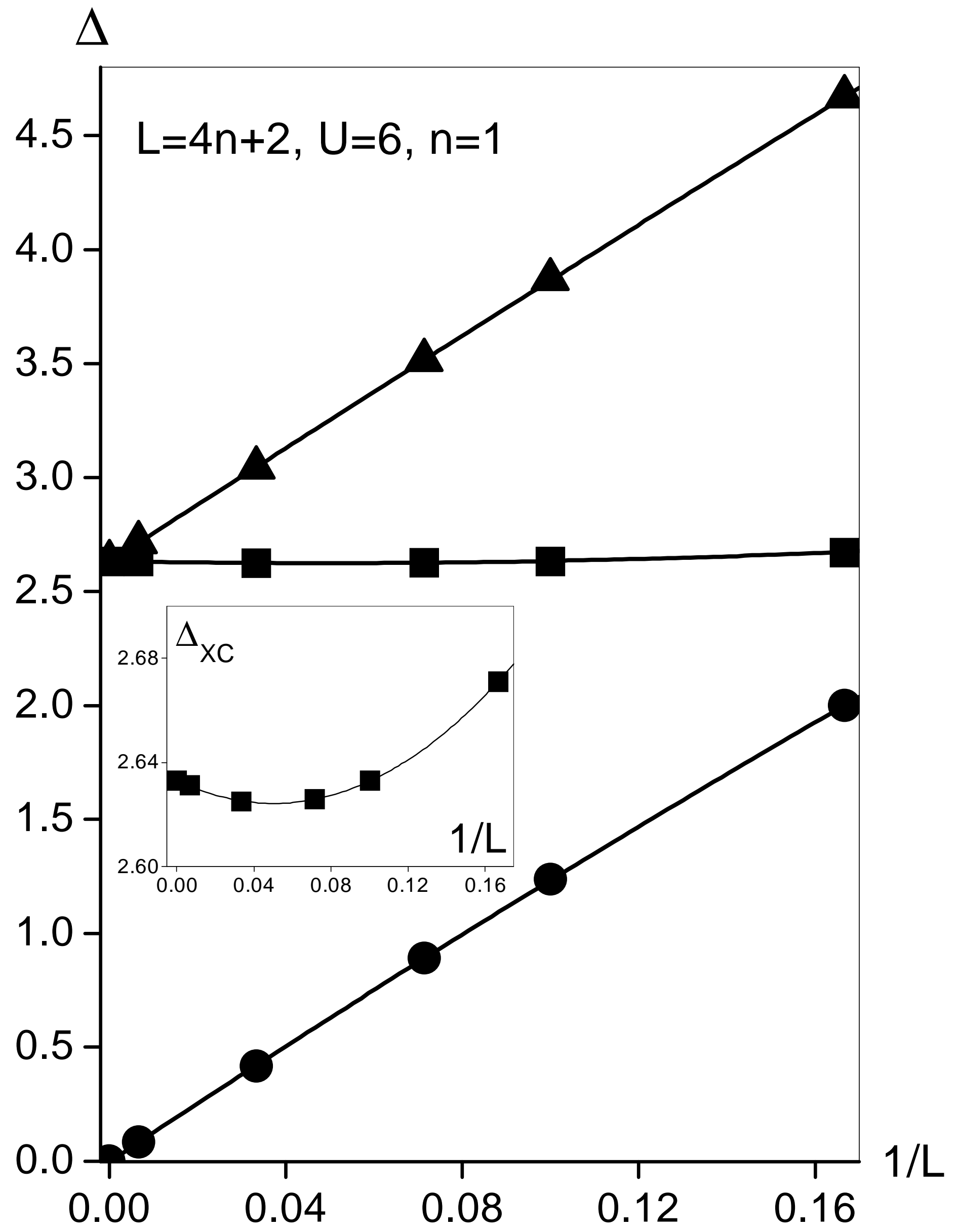

